\title{
Ceratocone: correlação entre grau evolutivo e padrão topográfico com o tipo de lente de contato adaptada
}

\author{
Keratoconus: correlation between degreesof severity and topographical pattern with \\ the kind offitted contact lens
}

\author{
Vinícius Coral Ghanem ${ }^{1}$ \\ Cleusa Coral-Ghanem ${ }^{2}$ \\ Ramon Coral Ghanem ${ }^{3}$ \\ Cristiane Larinho ${ }^{4}$
}

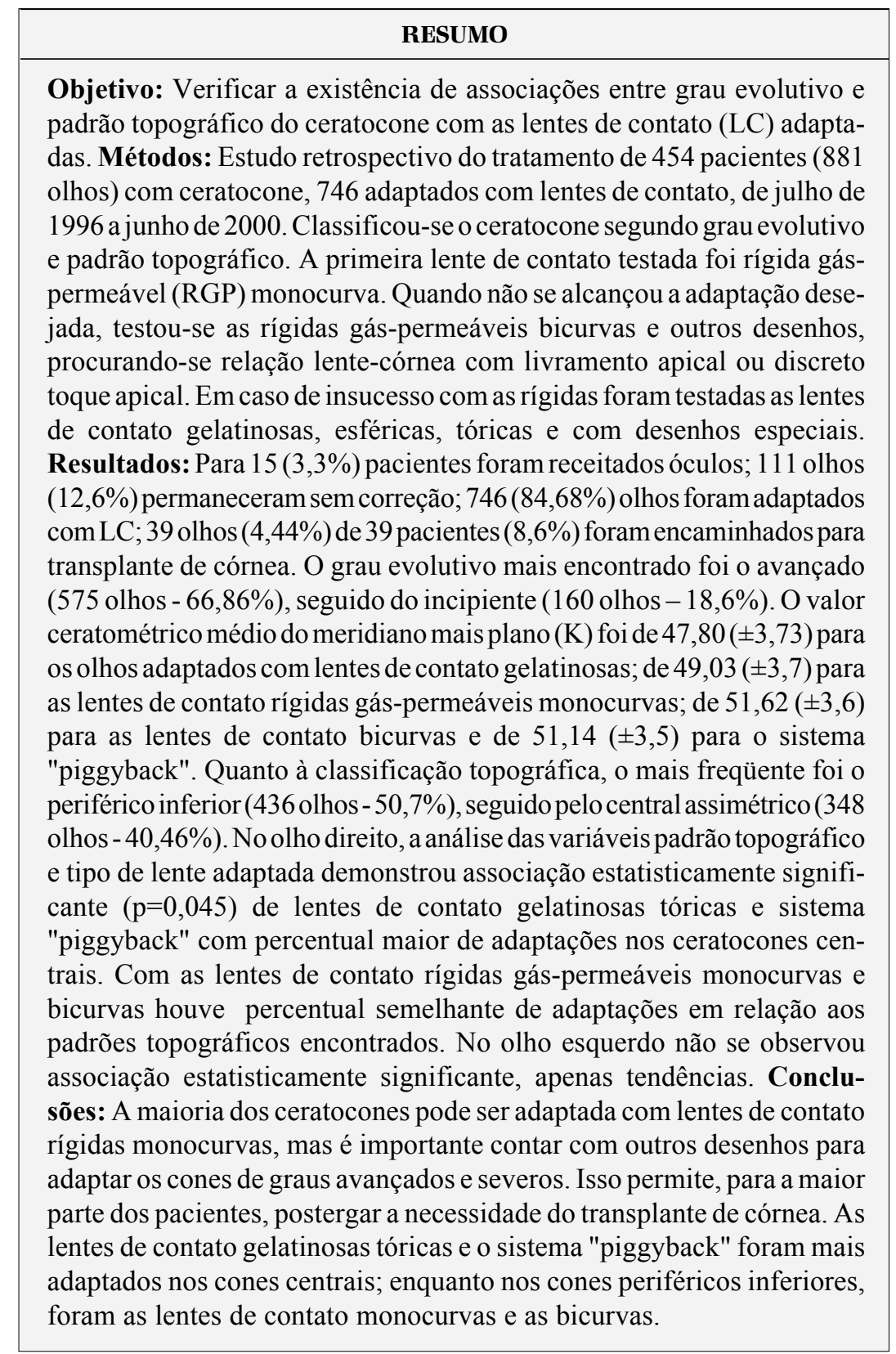

Descritores: Ceratocone/patologia; Acomodação ocular; Lentes de contato, Ceratoplastia penetrante; Lentes de contato hidrofílicas; Topografia da córnea

Objetivo: Verificar a existência de associações entre grau evolutivo e padrão topográfico do ceratocone com as lentes de contato (LC) adaptadas. Métodos: Estudo retrospectivo do tratamento de 454 pacientes ( 881 olhos) com ceratocone, 746 adaptados com lentes de contato, de julho de e padrão topográfico. A primeira lente de contato testada foi rígida gáspermeável (RGP) monocurva. Quando não se alcançou a adaptação deseprocurando-se relação lente-córnea com livramento apical ou discreto toque apical. Em caso de insucesso com as rígidas foram testadas as lentes Resultados: Para 15 (3,3\%) pacientes foram recitados óc 111 olhos (12,6\%) permaneceram sem correção; $746(84,68 \%)$ olhos foram adaptados com LC; 39 olhos $(4,44 \%)$ de 39 pacientes $(8,6 \%)$ foram encaminhados para transplante de córnea. $\mathrm{O}$ grau evolutivo mais encontrado foi o avançado (575 olhos - 66,86\%), seguido do incipiente ( 160 olhos $-18,6 \%$ ). O valor ceratométrico médio do meridiano mais plano $(\mathrm{K})$ foi de $47,80( \pm 3,73)$ para os olhos adaptados com lentes de contato gelatinosas; de 49,03 $( \pm 3,7)$ para as lentes de contato rígidas gás-permeáveis monocurvas; de $51,62( \pm 3,6)$ "piggyback". Quanto à classificação topográfica, o mais freqüente foi o periférico inferior (436 olhos - 50,7\%), seguido pelo central assimétrico (348 olhos - 40,46\%). No olho direito, a análise das variáveis padrão topográfico cante $(p=0,045)$ de lentes de contato gelatinosas tóricas e sistema "piggyback" com percentual maior de adaptações nos ceratocones cenbicurvas houve percentual semelhante de adaptações em relação aos padrões topográficos encontrados. No olho esquerdo não se observou associação estatisticamente significante, apenas tendências. Conclusões: A maioria dos ceratocones pode ser adaptada com lentes de contato rígidas monocurvas, mas é importante contar com outros desenhos para adaptar os cones de graus avançados e severos. Isso permite, para a maior parte dos pacientes, postergar a necessidade do transplante de córnea. As lentes de contato gelatinosas toricas e o sistema "piggyback" foram mais adaptados nos cones centrais; enquanto nos cones periféricos inferiore foram as lentes de contato monocurvas e as bicurvas.

\footnotetext{
Trabalho realizado no Departamento de Lentes de Contato do Hospital de Olhos Sadalla Amin Ghanem Joinville/SC

'Fellow do Departamento de Córnea e Doenças Externas da Universidade da Califórnia, Davis - EUA

${ }^{2}$ Médica Oftalmologista, Responsável pelo Setor de Lentes de Contato do Hospital de Olhos Sadalla Amin Ghanem. - Joinville/SC; pós-graduanda em nível de doutoramento pela Universidade de São Paulo (USP); ex-presidente da Sociedade Brasileira de Lentes de Contato e Córnea (SOBLEC)

${ }_{3}^{3}$ Médico Residente do Segundo Ano de Oftalmologia da

Universidade de São Paulo - USP

${ }^{4}$ Ortoptista do Hospital de Olhos Sadalla Amin Ghanem - Joinville/SC

Endereço para correspondência: Rua Fernando de Noronha, 225 apto. 901 - Joinville (SC) CEP 89203-070

E-mail: coral@coral-lentes.com.br

Recebido para publicação em 05.12.2002

Aceito para publicação em 22.07.2002
} 


\section{INTRODUÇÃO}

Ceratocone é uma ectasia corneal não inflamatória progressiva, na qual a córnea central ou paracentral assume forma cônica, permanecendo a periferia relativamente normal. A protrusão apical causa astigmatismo irregular, afilamento do ápice e a progressão da doença leva à formação de cicatrizes e importante baixa visual ${ }^{(1)}$. As lentes de contato (LC) rígidas são a principal opção para o tratamento de ceratocone porque tornam a superfície refrativa regular, neutralizam melhor do que qualquer outra as aberrações ópticas e as distorções da superfície corneal anterior, melhorando a visão, mesmo em graus avançados da doença ${ }^{(2-4)}$.

Os avanços tecnológicos dos desenhos e materiais das LC têm permitido sua adaptação em quase todos os graus de ceratocone. Além das rígidas gás-permeáveis (RGP) (esféricas, asféricas, zona óptica esférica com periferia asférica, bicurvas tipo Soper; tricurvas tipo $\mathrm{Ni}$-cone, policurvas tipo McGuire e outras com diferentes desenhos), pode-se utilizar LC gelatinosas (LCG) (esféricas, tóricas e desenhos especiais) e híbridas (tipo Softperm) ${ }^{(5-6)}$. Com o desenvolvimento das LC RGP de alto DK, o sistema "piggyback" (LC RGP de alto DK sobre LCG) tem sido indicado com maior freqüência. Suas funções são aliviar o desconforto, melhorar o posicionamento e auxiliar a estabilidade da $\mathrm{LC}$ rígida ${ }^{(7)}$, além de proteger o ápice nos casos de erosão recorrente, freqüente nos cones centrais em forma de bico ("nipple cone").

Ainda não existe consenso sobre qual a melhor forma de adaptação das LC no ceratocone, considerando seus diferentes padrões topográficos e graus evolutivos.

O objetivo deste estudo é verificar a existência de associação entre grau evolutivo e padrão topográfico do ceratocone com as LC adaptadas.

\section{MÉTODOS}

Estudo retrospectivo do tratamento de 454 pacientes portadores de ceratocone, novos ou já em acompanhamento, que procuraram o Departamento de Lentes de Contato do Hospital de Olhos Sadalla Amin Ghanem, no período compreendido entre julho de 1996 a junho de 2000.

Foram incluídos pacientes de todas as idades, independente de sexo e do uso anterior de LC, mas que tiveram acompanhamento mínimo de 3 meses até o final do estudo. O diagnóstico de ceratocone foi baseado em critérios já estabelecidos, incluídos nos sinais clínicos refrativos (reflexo retinoscópico em tesoura, aumento de miopia e astigmatismo irregular); biomicroscópicos (estrias de Vogt, anel de Fleischer, aumento da visibilidade das fibras nervosas, afinamento corneal e cicatrizes); ceratométricos (falta de paralelismo das miras e miras distorcidas); e videoceratoscópicos ${ }^{(8)}$ (Método de Rabinowitz McDonnell: poder da córnea central > 48,7 D, diferença entre a região corneal paracentral superior e inferior (valor I-S) > 1,4 D).

Foram excluídos do estudo portadores de doenças ocula- res ou sistêmicas com contra-indicações para o uso de LC, além dos pacientes já submetidos a transplante de córnea em ambos os olhos.

O ceratocone, segundo seu grau evolutivo foi classificado em 4 grupos $^{(9)}$ : incipiente (leituras ceratométricas menores que 45,00 dioptrias (D) nos dois meridianos); moderado (leituras entre 45,00 e 52,00 D nos dois meridianos); avançado (leituras entre 52,00 e 62,00 D nos dois meridianos) e severo (leituras maiores que $62,00 \mathrm{D}$ nos dois meridianos). Neste estudo, quando os 2 meridianos não se encontraram dentro do mesmo grupo, classificou-se o ceratocone pelo meridiano mais curvo. Quanto à aparência videoceratoscópica, a classificação utilizada foi de acordo com a localização do ápice do cone ${ }^{(10)}$ : central simétrico, central assimétrico, central indefinido, periférico superior, periférico inferior e periférico indefinido.

Pacientes não usuários de LC foram submetidos aos testes de adaptação quando a acuidade visual (AV) não era satisfatória com óculos. Os já usuários foram avaliados e, muitas vezes, readaptados. Todas as adaptações foram realizadas pelo mesmo autor (CCG).

Para determinar o sucesso da adaptação levou-se em conta: a relação lente-córnea pelo padrão fluoresceínico, posicionamento e mobilidade, visão satisfatória, tempo de uso aceitável com conforto e mínimas alterações do tecido corneal. Como as necessidades visuais e expectativas dos pacientes são muito variáveis, a definição de insucesso na adaptação foi considerada quando: 1)o tempo de uso com conforto era pequeno, incompatível com as necessidades do paciente, 2) AV insatisfatória para as necessidades individuais e 3)intolerância ao uso das LC. Os pacientes que tinham ceratocone avançado e severo, sem sucesso com a adaptação das LC, foram encaminhados para transplante de córnea.

As LC disponíveis para teste eram: LCG esféricas, tóricas e de desenhos especiais para ceratocone; LC RGP monocurvas, multi-esféricas e asféricas; rígidas tóricas e bicurvas (tipo Soper-McGuire).

No ceratocone incipiente, a primeira LC de teste foi RGP monocurva, com curva base igual a $\mathrm{K}$ (meridiano mais plano da córnea), acrescida de metade do astigmatismo ceratométrico; diâmetro de 9,0 $\mathrm{mm}$. Em ceratocone moderado, de 45 a $48 \mathrm{D}$, a primeira LC foi monocurva, com curva base igual a K, acrescida de um terço do astigmatismo ceratométrico; diâmetro de $8,6 \mathrm{~mm}$. Já no ceratocone moderado, de 48,50 a $52 \mathrm{D}$, a primeira LC foi monocurva com curva base em $\mathrm{K}$; diâmetro $8,3 \mathrm{~mm}$. No ceratocone avançado, a primeira LC de teste foi monocurva com curva base $1 \mathrm{D}$ mais plana do que K ; diâmetro de $8,0 \mathrm{~mm}$. Em ceratocone severo, a primeira LC de teste foi monocurva com curva base $2 \mathrm{D}$ mais plana do que $\mathrm{K}$; diâmetro igual ou menor do que $8,0 \mathrm{~mm}$.

Nos ceratocones moderado, avançado e severo, quando não se alcançou a adaptação desejada com a monocurva, testou-se a bicurva, tipo Soper modificado, com diâmetro em torno de 9,2 $\mathrm{mm}$; policurva e/ou desenhos especiais.

O diâmetro da LC dependeu muito do tipo de cone. Em cones redondos, centrais, utilizou-se diâmetros pequenos 
(de 7,5 a 8,5 mm); em cones ovais e excêntricos, diâmetros maiores (de 9 a 9,4 mm).

Procurou-se conseguir uma relação lente-córnea com livramento apical ou discreto toque apical, preferencialmente em três toques, objetivos muitas vezes não atingidos.

Nos casos de insucesso com as RGP e LCG, utilizou-se o sistema "piggyback" e, raramente, a LC híbrida (tipo Softperm).

A maioria dos pacientes necessitou de, pelo menos, três trocas de LC por olho, até se conseguir a adaptação desejada. Foram acompanhados por mais de 7 dias, antes da prescrição final.

Pacientes que não toleraram o uso de $\mathrm{LC}$ e aqueles com ceratocone avançado ou severo que apresentavam cicatrizes corneais axiais e baixa visual, incompatível com suas necessidades, foram encaminhados para ceratoplastia penetrante.

\section{Análise Estatística}

Para verificar a existência de associação entre as variáveis Grau Evolutivo e Padrão Topográfico com relação ao Tipo de Lente Adaptada, foi utilizado o teste Qui-quadrado. Quando os valores esperados eram menores que 5 , utilizou-se o teste exato de Fisher, tendo sido considerada significativa a associação quando o p-valor $\leq 0,05$. Os testes foram aplicados separadamente no Olho Direito e no Olho Esquerdo.

Devido à baixa freqüência de algumas categorias presentes nas variáveis, para análise estatística da associação entre Padrão Topográfico do ceratocone e Tipo de LC adaptada, considerou-se somente as variáveis: 1) Padrão Topográfico: central (simétrico e assimétrico), periférico inferior e periférico superior; e 2) Tipo de Lente adaptada: RGP monocurva, RGP bicurva, LCG tórica e sistema "piggyback". Para avaliar a associação entre Grau Evolutivo e Tipo de Lente adaptada, considerou-se as variáveis: 1) Grau Evolutivo: incipiente, moderado, avançado e severo; e 2) Tipo de Lente adaptada: RGP monocurva, RGP bicurva e sistema "piggyback".

Quando se trabalhou com a variável Grau Evolutivo (contínua) para comparar com o Tipo de Lente adaptada, optou-se pelo método das equações de Estimação Generalizada (EEG).

\section{RESULTADOS}

Durante o período do estudo, 881 olhos de 454 pacientes com ceratocone foram avaliados e classificados seguindo os critérios de inclusão. Eram 224 (49,3\%) do sexo feminino e 230 $(50,7 \%)$ do sexo masculino. Em relação à idade, dividiu-se em 3 grupos: a) 189 (41,6\%) tinham entre 10-25 anos, 208 (45,8\%) entre $26-40$ anos e $57(12,6 \%)$ acima de 41 anos. A idade média foi de 29,03 anos $( \pm 9,93)$ variando de 10 a 70 anos. Ceratocone unilateral foi encontrado em 27 pacientes $(5,95 \%)$.

Quanto à forma de tratamento, para $15(3,3 \%)$ pacientes foram receitados óculos; 111 olhos (12,6\%) permaneceram sem correção; $746(84,68 \%)$ olhos foram adaptados com LC; 39 olhos $(4,44 \%)$ de 39 pacientes $(8,6 \%)$ foram encaminhados para transplante de córnea; desses, $21(2,4 \%)$ foram submeti- dos à cirurgia e os 18 restantes $(2,04 \%)$ continuavam na lista de espera até o final do estudo.

Os resultados referentes a grau evolutivo, padrão topográfico e tipo de LC adaptada estão dispostos nas Tabelas 1,2 e 3 , respectivamente. $\mathrm{O}$ grau evolutivo mais encontrado foi o Avançado em 575 olhos (65,27\%), seguido do Incipiente em 160 olhos $(18,16 \%)$. Quanto à classificação topográfica, o tipo Periférico Inferior foi o mais encontrado, em 436 olhos (49,49\%), seguido pelo Central Assimétrico em 348 olhos $(39,50 \%)$. Sem considerar as formas Indefinidas, o menos freqüente foi o tipo Periférico Superior em 24 olhos (2,72\%). Dos tipos de LC, as mais freqüentemente adaptadas foram as RGP monocurvas em 529 olhos (60,04\%), seguidas pelas RGP bicurvas, tipo Soper - McGuire, em 113 olhos (12,83\%).

Ao se considerar os valores ceratométricos, observa-se que a média das ceratometrias do meridiano mais plano $(\mathrm{K})$ dos olhos adaptados com LCG (esféricas, tóricas ou com desenhos especiais para ceratocone) foi de $47,80( \pm 3,73)$, variando de 43,00 a 60,75 D; com LC RGP monocurva foi de 49,03 $( \pm 3,7)$, variando de 42,50 a $69,00 \mathrm{D}$; com LC RGP bicurva foi de

\begin{tabular}{|lr|}
\hline \multicolumn{2}{|c|}{ Tabela 1. Classificação do ceratocone segundo seus graus evolutivos } \\
Classificação & $\mathbf{n}(\%)$ \\
Incipiente & $160(18,60 \%)$ \\
Moderado & $98(11,39 \%)$ \\
Avançado & $575(66,86 \%)$ \\
Severo & $27(3,14 \%)$ \\
Total & 860 \\
$\mathrm{n}=$ número de olhos & \\
\hline
\end{tabular}

\begin{tabular}{|c|c|}
\hline \multicolumn{2}{|c|}{$\begin{array}{l}\text { Tabela 2. Classificação do ceratocone segundo aparência } \\
\text { topográfica }\end{array}$} \\
\hline Classificação & n (\%) \\
\hline Central assimétrico & $348(40,46 \%)$ \\
\hline Central indefinido & $2(0,23 \%)$ \\
\hline Central simétrico & $38(4,42 \%)$ \\
\hline Periférico indefinido & 12 ( $1,39 \%)$ \\
\hline Periférico inferior & $436(50,7 \%)$ \\
\hline Periférico superior & 24 ( $2,8 \%)$ \\
\hline Total & 860 \\
\hline $\mathrm{n}=$ número de olhos & \\
\hline
\end{tabular}

\begin{tabular}{|lr|}
\hline \multicolumn{2}{|c|}{ Tabela 3. Tipo de Lente de Contato Adaptada } \\
Tipo de LC & $\mathbf{n}(\%)$ \\
LCG esféricas & $14(1,88 \%)$ \\
LCG tóricas & $25(3,35 \%)$ \\
LC rígidas monocurva & $529(70,91 \%)$ \\
RGP tórica & $18(2,41 \%)$ \\
Rígida bicurva & $113(15,15 \%)$ \\
Sistema "piggyback" & $33(4,42 \%)$ \\
Outras (LCG com desenhos & $14(1,88 \%)$ \\
especiais, Softperm e Millenium) & $746(100 \%)$ \\
Total & 740 lánel \\
LCG = lente de contato gelatinosa; RGP= rígida gás-permeável
\end{tabular}


$51,62( \pm 3,6)$, variando de 43,50 a $61,50 \mathrm{D}$ e com sistema "piggyback", de $51,14( \pm 3,5)$, variando de 44,50 a $58,00 \mathrm{D}$.

A análise das variáveis Padrão Topográfico e Tipo de Lente adaptada demonstrou, no Olho Direito, associação estatisticamente significante $(p=0,045)$ : 1$)$ LCG tóricas e sistema "piggyback" tiveram um percentual maior de adaptações em cones centrais; 2) LCG tóricas, em cones superiores. Com as LC RGP monocurvas e bicurvas, o percentual de adaptações foi semelhante, em relação aos padrões topográficos estudados (Tabela 4). No Olho Esquerdo não se observou associação estatisticamente significante $(\mathrm{p}=0,065)$, mas verificou-se uma leve tendência: 1) LCG tóricas, RGP bicurvas e sistema "piggyback" tiveram um percentual maior de adaptações nos cones centrais; e 2) LC RGP monocurvas, nos cones inferiores (Tabela 5).

A análise das variáveis Grau Evolutivo e Tipo de Lente adaptada demonstrou, no Olho Direito, não haver associação estatisticamente significante $(\mathrm{p}=0,066)$, apenas uma leve tendência: 1$)$ nos graus Incipiente e Moderado houve um maior percentual de adaptações com as RGP monocurvas; 2) no grau evolutivo Avan- çado, com o sistema "piggyback"; 3) nos graus evolutivos Avançado e Severo, com as RGP bicurvas (Tabela 6). No Olho Esquerdo observou-se significância estatística $(p=0,001)$ : 1$)$ nos graus Incipiente e Moderado, com as RGP monocurvas; 2) no grau Avançado, com o sistema "piggyback"; e 3) nos graus Avançado e Severo, com as RGP bicurvas (Tabela 7).

Ao se analisarem os resultados do Modelo pelo método das EEG, observou-se que os pacientes que utilizavam as RGP monocurvas, bicurvas ou o sistema "piggyback" apresentavam ceratocone mais avançado, principalmente os olhos adaptados com LC bicurva ou com o sistema "piggyback". Não se observou diferença no grau evolutivo com relação ao olho afetado (Tabela 8). As demais variáveis não foram significativas.

A AV média dos usuários de LC foi de $20 / 35( \pm 16,75)$, variando de $20 / 20$ a $20 / 200$, sendo que $86,5 \%$ dos olhos alcançaram 20/40 ou mais (Tabela 9).

\section{DISCUSSÃO}

As LC foram a melhor forma de tratamento em $746(84,68 \%)$

Tabela 4. Análise da associação entre as aparências topográficas dos ceratocones e os tipos de lentes de contato adaptados no olho direito

\begin{tabular}{|c|c|c|c|c|c|}
\hline \multirow[t]{2}{*}{ Aparências topográficas } & \multicolumn{5}{|c|}{ Tipos de lentes de contato } \\
\hline & $\begin{array}{c}\text { LCG tórica } \\
\text { n (\%) }\end{array}$ & $\begin{array}{c}\text { Monocurva } \\
\text { n (\%) }\end{array}$ & $\begin{array}{c}\text { "Piggyback" } \\
\text { n (\%) }\end{array}$ & $\begin{array}{c}\text { Bicurva } \\
\text { n (\%) }\end{array}$ & $\begin{array}{l}\text { Total } \\
\text { n (\%) }\end{array}$ \\
\hline Central & $10(76,92 \%)$ & $126(44,68 \%)$ & $14(66,67 \%)$ & $21(46,67 \%)$ & $171(47,37 \%)$ \\
\hline Periférico inferior & $2(15,38 \%)$ & $150(53,19 \%)$ & $7(33,33 \%)$ & $23(51,11 \%)$ & $182(50,42 \%)$ \\
\hline Periférico superior & $1(7,69 \%)$ & $6(2,13 \%)$ & $0(0 \%)$ & $1(2,22 \%)$ & $8(2,22 \%)$ \\
\hline Total & 13 & 282 & 21 & 45 & 361 \\
\hline Teste Exato de Fischer (bicaudal) & $p=0,045$ & & & & \\
\hline LCG $=$ Lente de contato gelatinosa & & & & & \\
\hline
\end{tabular}

Tabela 5. Análise da associação entre as aparências topográficas dos ceratocones e os tipos de lentes de contato adaptadas no olho esquerdo

\begin{tabular}{|c|c|c|c|c|c|c|}
\hline \multirow{2}{*}{ Aparências topográficas } & \multicolumn{6}{|c|}{ Tipos de lentes de contato } \\
\hline & $\begin{array}{c}\text { LCG tórica } \\
\text { n (\%) }\end{array}$ & $\begin{array}{c}\text { Monocurva } \\
\text { n (\%) }\end{array}$ & $\begin{array}{c}\text { "Piggyback" } \\
\text { n (\%) }\end{array}$ & $\begin{array}{c}\text { Bicurva } \\
\text { n (\%) }\end{array}$ & & $\begin{array}{l}\text { Total } \\
\text { n (\%) }\end{array}$ \\
\hline Central & $6(66,67 \%)$ & $87 \quad(37,18 \%)$ & $7 \quad(63,64 \%)$ & $36(54,55 \%)$ & 136 & $(42,50 \%)$ \\
\hline Periférico superior & $0(0 \%)$ & $6(2,56 \%)$ & $0(0 \%)$ & $2(3,03 \%)$ & 8 & $(2,50 \%)$ \\
\hline Total & 9 & 234 & 11 & 66 & 320 & \\
\hline Teste Exato de Fischer (bicaudal) & $p=0,065$ & & & & & \\
\hline $\mathrm{LCG}=$ Lente de contato gelatinosa & & & & & & \\
\hline
\end{tabular}

Tabela 6. Análise da associação entre os graus evolutivos do ceratocone e os tipo de lentes de contato adaptadas no olho direito

\begin{tabular}{|c|c|c|c|c|c|}
\hline \multirow[t]{2}{*}{ Graus evolutivos } & \multicolumn{5}{|c|}{ Tipos de lentes de contato } \\
\hline & $\begin{array}{c}\text { Monocurva } \\
\text { n (\%) }\end{array}$ & $\begin{array}{c}\text { "Piggyback" } \\
\text { n (\%) }\end{array}$ & $\begin{array}{c}\text { Bicurva } \\
\text { n (\%) }\end{array}$ & & $\begin{array}{l}\text { Total } \\
\text { n (\%) }\end{array}$ \\
\hline Incipiente & $30 \quad(10,49 \%)$ & 1 ( $4,76 \%)$ & $1 \quad(2,22 \%)$ & 32 & $(9,09 \%)$ \\
\hline Moderado & $198(69,23 \%)$ & $13(61,90 \%)$ & $28(62,22 \%)$ & 239 & $(67,90 \%)$ \\
\hline Avançado & $53(18,53 \%)$ & 7 (33,33\%) & $13(28,89 \%)$ & 73 & $(20,74 \%)$ \\
\hline Severo & $5(1,75 \%)$ & $0 \quad(0 \%)$ & $3(6,67 \%)$ & 8 & ( $2,27 \%)$ \\
\hline Total & 282 & 21 & 45 & 361 & \\
\hline Teste Exato de Fischer (bicaudal) & & & & & \\
\hline
\end{tabular}




\begin{tabular}{|c|c|c|c|c|}
\hline \multirow{2}{*}{ Graus evolutivos } & \multicolumn{4}{|c|}{ Tipos de lentes de contato } \\
\hline & $\begin{array}{c}\text { Monocurva } \\
\text { n (\%) }\end{array}$ & $\begin{array}{c}\text { "Piggyback" } \\
\text { n (\%) }\end{array}$ & $\begin{array}{c}\text { Bicurva } \\
\text { n (\%) }\end{array}$ & $\begin{array}{l}\text { Total } \\
\text { n (\%) }\end{array}$ \\
\hline Incipiente & $25(10,46 \%)$ & $0(0 \%)$ & $1(1,47 \%)$ & $26(8,15 \%)$ \\
\hline Moderado & $175(73,22 \%)$ & $7 \quad(58,33 \%)$ & $34(50,00 \%)$ & $216(67,71 \%)$ \\
\hline Avançado & $37(15,48 \%)$ & $5 \quad(41,67 \%)$ & $27(39,71 \%)$ & $69(21,63 \%)$ \\
\hline Severo & $2(0,84 \%)$ & $0(0 \%)$ & $6(8,82 \%)$ & $8(2,51 \%)$ \\
\hline Total & 239 & 12 & 68 & 319 \\
\hline Teste Exato de Fischer (bicaudal) & & & & \\
\hline
\end{tabular}

dos 881 olhos com ceratocone avaliados neste estudo, sendo reconhecidamente a melhor forma de tratamento para pacientes com ceratocone ${ }^{(11)}$.

Devido às variações acentuadas na topografia corneal dos pacientes com ceratocone, um tipo único de LC não proporciona uma adaptação ideal para todos os casos.

As primeiras tentativas de adaptação foram realizadas com as LC monocurvas, explicando a elevada freqüência de seu uso; concordante com dados de Crews et al ${ }^{(12)}$. Quando não se conseguiu boa relação lente-córnea com as LC monocurvas, foram testadas as LC bicurvas, procurando-se obter padrão fluoresceínico com livramento apical ou toque central mínimo. Essa filosofia de adaptação fez com que os ceratocones incipientes e moderados fossem mais freqüentemente adaptados com as LC monocurvas. Nos graus avançados e severos, onde geralmente o toque central é acentuado com as LC monocurvas, foram testadas, também, as LC bicurvas e, em casos especiais, o sistema "piggyback". O desenho das LC rígidas bicurvas baseia-se no aumento da abóbada sagital conseguido por meio da diminuição do raio da curva central posterior ou pelo aumento do diâmetro da zona óptica central, facilitando a adaptação em cones avançados e severos, como observado neste estudo. O sistema "piggyback" foi mais utilizado em casos de erosão recorrente de ápice de cone, para aliviar o desconforto e permitir maior tempo de uso. Tsubota $\mathrm{K}$ et $\mathrm{al}^{(7)}$ relataram que $90,9 \%$ dos pacientes com ceratocone avançado ou severo que não podiam tolerar LC rígidas foram capazes de evitar a intervenção cirúrgica com o uso do sistema "piggyback". Já em nosso estudo, observou-se que os cones severos foram adaptados com lentes monocurvas e principalmente as bicurvas; em nenhum caso foi utilizado o sistema "piggyback", o qual apresentou associação com as adaptações em ceratocones avançados.

Ao se analisarem as variáveis Padrão Topográfico e Tipo de Lente adaptada, observou-se que as únicas associações

\begin{tabular}{|lcr|}
\hline \multicolumn{3}{|c|}{$\begin{array}{c}\text { Tabela 8. Parâmetros estimados no modelo final pelo método } \\
\text { das equações de estimação generalizada }\end{array}$} \\
Parâmetro & Estimativa & p-valor \\
Intercepto & 47,6939 & 0,0001 \\
Monocurva & 1,5059 & 0,0001 \\
"Piggyback" & 3,1659 & 0,0002 \\
Bicurva & 3,4175 & 0,0001 \\
Olho D/E & $-0,0546$ & 0,7857 \\
D = direito E = esquerdo & & \\
\hline
\end{tabular}

encontradas no olho direito e no esquerdo foram as adaptações das LCG tóricas e do sistema "piggyback" nos cones centrais. As outras associações não foram encontradas em nenhum dos olhos, sugerindo que não sejam verdadeiras.

As LCG podem fornecer visão aceitável e conforto para pacientes com ceratocone, provando ser um auxílio visual útil. A AV é mais satisfatória em cones de grande diâmetro do que em cones centrais de pequeno diâmetro ${ }^{(6)}$. As LCG tóricas ficaram mais bem adaptadas nos cones centrais, possivelmente por ter a córnea, com este tipo de cone, uma configuração mais semelhante ao desenho padrão da LC, facilitando o posicionamento e a estabilidade do eixo. Deve-se lembrar que para a adaptação no ceratocone, as LCG não são a primeira opção porque os resultados ópticos são inferiores aos das LC rígidas. O uso do sistema "piggyback" está associado com os cones centrais, uma vez que a LCG protege o ápice nos casos de erosão recorrente, freqüentemente encontrada nos tipos de cone em forma de bico.

Uma das mais importantes inovações para os cones inferiores foi o desenvolvimento de LC com zona óptica descentrada inferiormente (Menicon Decentered $\mathrm{OZ}^{\circledR}$ ), que permite mais estabilidade e melhor visão ${ }^{(13)}$. Pela ausência, no mercado brasileiro, de desenhos especiais para cones periféricos, utilizou-se monocurvas esféricas ou asféricas de grande diâmetro, apesar de provocarem toque acentuado sobre o ápice do cone. Essa relativa falta de opção fez com que as LC RGP monocurvas tenham sido as mais adaptadas nesses casos.

As LC bicurvas tipo Soper e desenhos derivados foram desenvolvidas para promover livramento do ápice do cone ou

\begin{tabular}{|cr|}
\hline $\begin{array}{c}\text { Tabela 9. Acuidade visual nos usuários de lentes de contato } \\
\text { Acuidade Visual }\end{array}$ & $\mathbf{n}(\%)$ \\
$20 / 20$ & $25(3,35 \%)$ \\
$20 / 25$ & $240(32,20 \%)$ \\
$20 / 30$ & $230(30,83 \%)$ \\
$20 / 40$ & $150(20,11 \%)$ \\
$20 / 50$ & $44(5,90 \%)$ \\
$20 / 60$ & $25(3,35 \%)$ \\
$20 / 70$ & $16(2,14 \%)$ \\
$20 / 80$ & $8(1,07 \%)$ \\
$20 / 100$ & $3(0,40 \%)$ \\
$20 / 150$ & $3(0,40 \%)$ \\
$20 / 200$ & $2(0,27 \%)$ \\
Total & 746 \\
\hline
\end{tabular}


permitir apenas discreto toque apical. Embora sejam mais indicadas para cones centrais, podem apresentar bons resultados em cones paracentrais, quando a posição do ápice não se encontra muito distante do centro óptico. Neste estudo, as LC bicurvas mostraram uma tendência de melhor adaptação nos cones centrais no olho esquerdo; associação não observada no olho direito, demonstrando que a relação entre LC bicurvas e cones centrais não é obrigatória.

Um dos fatores que determina o sucesso da adaptação é a AV alcançada com as LC. Semelhante a este estudo, outros também relataram que, em média, $80 \%$ dos olhos atingem $\mathrm{AV}$ melhor ou igual a $20 / 40^{(2,14)}$, muito satisfatória para a maioria dos pacientes.

Muitos trabalhos ${ }^{(3,14-17)}$ mostram que apenas 10 a $20 \%$ dos pacientes com ceratocone necessitam de transplante de córnea. Estudos têm confirmado que, na maioria dos pacientes, a ceratoplastia penetrante pode ser protelada ou evitada pela adaptação de $\mathrm{LC}^{(11,17-18)}$.

No estudo de Crews MJ et al. ${ }^{(12)}, 29 \%$ de 123 olhos foram encaminhados para transplante e, no de Lass et al ${ }^{(14)}, 21 \%$ de 746 olhos. Acreditamos que esses valores altos não sejam concordantes com a realidade da prática oftalmológica fora dos serviços de referência, para onde são encaminhados pacientes com quadros mais avançados. Além disso, é difícil comparar o percentual entre as diversas publicações, pois os parâmetros de AV utilizados para encaminhamento ao transplante diferem, variando de $20 / 50^{1(4)}$ a $20 / 100^{(2)}$. Em nosso estudo, 96 pacientes $(21,14 \%)$ apresentaram $\mathrm{AV}$, com LC, igual ou inferior a 20/60. Desses, $57(12,56 \%)$ consideraram a visão satisfatória e preferiram continuar com LC. Os outros 39 $(8,59 \%)$ foram encaminhados para transplante de córnea. Desses, $21(2,4 \%)$ foram submetidos à cirurgia e os $18(2,04 \%)$ restantes continuavam na lista de espera até o final do estudo.

Nossa baixa indicação para transplante deve-se, provavelmente, a múltiplas opções de desenhos de $\mathrm{LC}$ e ao fato de modificarmos as LC rígidas no consultório, alterando o desenho padrão, aumentando o número de curvas, abrindo as periféricas e/ou reduzindo o diâmetro, de acordo com cada caso. Além disso, encaminhamos três pacientes para a remoção da nébula no ápice do cone em bico, com Excimer Laser. Dois meses após, os três pacientes voltaram a usar LC sem desconforto e com melhor AV.

\section{CONCLUSÃo}

Baseando-se nos dados deste estudo, conclui-se que as LC monocurvas adaptam com sucesso a maioria dos ceratocones, embora nos casos avançados e severos é importante a disponibilidade de outros desenhos, principalmente as LC bicurvas e o sistema "piggyback". Isso permite maior número de adaptações, postergando a necessidade do transplante de córnea. As LCG tóricas e o sistema "piggyback" foram mais adaptados nos cones centrais; enquanto nos cones periféricos inferiores, foram as LC monocurvas e as bicurvas.

\section{ABSTRACT}

Purpose: To determine associations between degrees of severity and topographical patterns of keratoconus with fitted contact lenses (CL). Methods: Retrospective study of 454 patients (881 eyes) with keratoconus, 746 fitted with contact lenses, from July 1996 to June 2000. Keratoconus was classified according to degrees of severity and topographical pattern. The first tested contact lens was the spherical rigid gas permeable. When it did not achieve the desired fitting, the bicurve rigid gas permeable and other designs were tried, in search of an apical clearance or slight apical touch fitting relationship. Results: For 15 (3.3\%) patients glasses were prescribed; 111 eyes (12.6\%) remained uncorrected; 746 $(84.68 \%)$ eyes were fitted with contact lenses; for 39 eyes $(4.44 \%)$ of 39 patients $(8.6 \%)$ penetrating keratoplasty was recommended. The most common degree of severity found was advanced ( 575 eyes $-66.86 \%$ ), followed by the mild variety $(160$ eyes $-18.6 \%)$. The average keratometric value of the flattest meridian $(\mathrm{K})$ was $47.80( \pm 3.73)$ for the eyes fitted with soft CL; $49.03( \pm 3.7)$ for the spherical rigid gas permeable contact lenses; of $51.62( \pm 3.6)$ for the bicurve contact lenses and $51.14( \pm 3.5)$ for the piggyback system. Concerning the topographical pattern, the most frequent was inferior peripheral cone ( 436 eyes $-50.7 \%$ ), followed by central asymmetrical cone ( 348 eyes $-40.46 \%$ ). In the right eye, the analysis of the variables, topographical pattern and kind of fitted lens showed a statistically significant association $(\mathrm{p}=0.045)$ of toric soft contact lenses and piggyback system with a higher percentage of fittings in central cone. Concerning the rigid spherical and bicurve lenses, the fitting percentage was similar in relation to the found topographical pattern. In the left eye, statistically significant associations were not observed, only tendencies. Conclusion: Spherical contact lenses can be fitted to the majority of patients with keratoconus, but it is important to rely on other designs to fit the advanced and severe cases. This allows most patients to postpone the need for corneal transplant. Toric soft contact lenses and piggyback system were more frequently fitted to central cones; whereas in inferior peripheral cones, spherical and bicurve contact lenses were those more used.

Keywords: Keratoconus/pathology; Ocular accommodation; Contact lenses; Penetrating keratoplasty, Hydrophilic contact lenses; Corneal topography

\section{REFERÊNCIAS}

1. Krachmer JH, Feder RS, Belin MW. Keratoconus and related non-inflammatory corneal thinning disorders. Surv Ophthalmol 1984,28:293-322.

2. Leça RG, Fukushima N, Gonzaga R, Lipener C, Zamboni F, Lewinsky R et al. Estudo clínico da adaptação de lentes de contato no ceratocone. Arq Bras Oftalmol 1995;58:149-51.

3. Fowler WC, Belin MW, Chambers WA. Contact lens in the visual correction of keratoconus. CLAO J 1988;14:203-6.

4. Griffiths M, Zahner K, Collins M, Carney L. Masking of irregular corneal topography with contact lenses. CLAO J 1998;24:76-81. 
5. Maguen E, Caroline P, Rosner IR, Macy J, Nesburn AB. The use of the SoftPerm lens for the correction of irregular astigmatism. CLAO J 1992;18:173-6.

6. Koliopoulos J, Tragakis M. Visual correction of keratoconus with soft contact lenses. Ann Ophthalmol 1981;13:835-7.

7. Tsubota K, Mashima Y, Murata H, Yamada M. A piggyback contact lens for the correction of irregular astigmatism in keratoconus. Ophthalmology 1994; 101:134-9.

8. Rabinowitz YS, McDonnell PJ. Computer-assisted corneal topography in keratoconus. Refract Corneal Surg 1989;5:400-8.

9. Buxton JN, Keattes RH, Hoefle FB, et al. The contact lens correction of keratoconus. In: Dabezies Jr. OH. The CLAO guide to basic science and clinical practice. Orlando: Grune \& Statton; 1984. p.55.1-55.14.

10. Wilson SE, Lin DTC, Klyce SD. Corneal topography of keratoconus. Cornea 1991;10:2-8

11. Kastl PR, Donzis PB, Cole HP, Rice J, Baldone JA. A 20-year retrospective study of the use of contact lenses in keratoconus. CLAO J 1987;13:102-4.
12. Crews MJ, Driebe Jr. WT, Stern GA. The clinical management of keratoconus: a 6 year retrospective study. CLAO J 1994;20:194-7.

13. Sakamoto OD, Miyanaga, MD. Descentered optic zone design for keratoconus. Spectrum 1997;30:41-4.

14. Lass JH, Lembach RG, Park SB, Hom DL, Fritz ME, Svilar GM, et al. Clinical management of keratoconus. A multicenter analysis. Ophthalmology 1990;97:433-45.

15. Kennedy RH, Bourne WM, Dyer JA. A 48-year clinical and epidemiologic study of keratoconus. Am J Ophthalmol 1986;101:267-73.

16. Mandell RB. Contemporary management of keratoconus. Int Contact Lens Clin 1997;24:43-58.

17. Smiddy WE, Hamburg TR, Kracher GP, Stark WJ. Keratoconus: contact lens or keratoplasty? Ophthalmology 1988;95;487-92.

18. Belin MW, Fowler WG, Chambers WA. Keratoconus: evaluation of recent trends in the surgical and nonsurgical correction of keratoconus. Ophthalmology 1988;95:335-9.

\section{Simpósio Internacional de Afualização em Oftalmologia da Santa Casa de São Paulo}

\section{5 a 7 de Junho de 2003}

Frei Caneca Shopping \& Convention Center São Paulo - SP

\section{Promoção \\ Centro de Estudos Oftalmológicos Jacques Tupinambá}

INFORMAC̣ÕES: JDE Comunicação e Eventos
Tels.: (1 1 ) 289-4301 / 287-9378
Fax: (11) 288-8157
E-mail: jdecomev@uol.com.br 